OPEN ACCESS

Edited by:

Fabio Marroni,

Istituto di Genomica Applicata, Italy

Reviewed by:

Yi Zheng,

Boyce Thompson Institute, USA

Sara Sestili,

Consiglio per la Ricerca in Agricoltura e l'Analisi dell'Economia Agraria, Italy

*Correspondence:

Qiusheng Kong

qskong@mail.hzau.edu.cn

Zhilong Bie

biezhilong@hotmail.com

Specialty section:

This article was submitted to Technical Advances in Plant Science, a section of the journal

Frontiers in Plant Science

Received: 28 June 2016

Accepted: 21 July 2016

Published: 03 August 2016

Citation:

Kong Q, Gao L, Cao L, Liu Y, Saba H, Huang $Y$ and Bie Z (2016) Assessment of Suitable Reference

Genes for Quantitative Gene Expression Studies in Melon Fruits.

Front. Plant Sci. 7:1178

doi: 10.3389/fpls.2016.01178

\section{Assessment of Suitable Reference Genes for Quantitative Gene Expression Studies in Melon Fruits}

\author{
Qiusheng Kong*, Lingyun Gao, Lei Cao, Yue Liu, Hameed Saba, Yuan Huang and \\ Zhilong Bie*
}

Key Laboratory of Horticultural Plant Biology, Ministry of Education; College of Horticulture and Forestry Sciences, Huazhong Agricultural University, Wuhan, China

Melon (Cucumis melo L.) is an attractive model plant for investigating fruit development because of its morphological, physiological, and biochemical diversity. Quantification of gene expression by quantitative reverse transcription polymerase chain reaction (qRTPCR) with stably expressed reference genes for normalization can effectively elucidate the biological functions of genes that regulate fruit development. However, the reference genes for data normalization in melon fruits have not yet been systematically validated. This study aims to assess the suitability of 20 genes for their potential use as reference genes in melon fruits. Expression variations of these genes were measured in 24 samples that represented different developmental stages of fertilized and parthenocarpic melon fruits by qRT-PCR analysis. GeNorm identified ribosomal protein $L$ (CmRPL) and cytosolic ribosomal protein S15 (CmRPS15) as the best pair of reference genes, and as many as five genes including CMRPL, CmRPS15, TIP41-like family protein (CmTIP41), cyclophilin ROC7 (CmCYP7), and ADP ribosylation factor 1 (CmADP) were required for more reliable normalization. NormFinder ranked CMRPS15 as the best single reference gene, and RAN GTPase gene family (CmRAN) and TATA-box binding protein (CmTBP2) as the best combination of reference genes in melon fruits. Their effectiveness was further validated by parallel analyses on the activities of soluble acid invertase and sucrose phosphate synthase, and expression profiles of their respective encoding genes CmAIN2 and CmSPS1, as well as sucrose contents during melon fruit ripening. The validated reference genes will help to improve the accuracy of gene expression studies in melon fruits.

Keywords: Cucumis melo, fruit development, gene expression, reference gene, normalization, qRT-PCR

\section{INTRODUCTION}

Melon (Cucumis melo L.) is a cucurbitaceous crop cultivated worldwide, and it is one of the most important fleshy fruits used for fresh consumption. Melon fruits exhibit extreme diversity in terms of shape, size, flesh, color, sweetness, aroma, and fruit texture (Nunez-Palenius et al., 2008). In addition, melon fruits have significant variations in their ripening physiology; the fruits can be categorized as either climacteric or non-climacteric types based on their ripening-related respiration rate and ethylene evolution profiles (Clepet et al., 2011; Leida et al., 2015; Saladié et al., 2015). These diverse traits can be exploited to reveal the underlying biological processes and 
mechanisms regulating fruit development. Accordingly, melon is considered an alternative model plant for elucidating fruit ripening (Ezura and Owino, 2008). Extensive molecular and genetic studies have been conducted on melon in recent years to understand the regulatory mechanisms of fruit development and ripening, with the aim of improving its fruit quality (Moreno et al., 2008; Dai et al., 2011; Portnoy et al., 2011; Vegas et al., 2013; Díaz et al., 2014; Saladié et al., 2015).

Gene expression data during melon fruit development is crucial to study the fruit expansion and maturity mechanisms. Quantitative reverse transcription polymerase chain reaction (qRT-PCR) has currently become the widely used method for quantification of target gene expression because of its sensitivity and rapidness. However, qRT-PCR is a multiple-step method and its use is inherently variable, which may cause the gene expression data to differ from the actual data (Nolan et al., 2006). To overcome this limitation of the technique and to ensure its accurate results, a robust normalization strategy is applied by using reference genes that have been shown to be stably expressed under the experimental conditions (Dheda et al., 2005; Huggett et al., 2005). Consequently, selection of reference genes with stable expression is a very important step prior to qRT-PCR analysis (Gutierrez et al., 2008).

The quantification of gene expressions in melon fruits by qRT-PCR have been conducted in several studies, and different reference genes were used for normalization, such as actin (ACT; Shan et al., 2012; Sun et al., 2013), cyclophilin (CYP; Gonzalez-Ibeas et al., 2007; Portnoy et al., 2011; Qin et al., 2011; Galpaz et al., 2013; Gonda et al., 2013), and glyceraldehyde-3phosphate dehydrogenase (GAPDH; Gao et al., 2013). However, the expression stability of these genes in melon fruits has not been systematically assessed to date. Validations of reference genes have been performed in roots, leaves, and stems of melons (Kong et al., 2014b; Sestili et al., 2014), but not in fruits and other organs. Reference genes have been proven to be organspecific or tissue-specific in several studies (Guénin et al., 2009; Kong et al., 2015). Therefore, the reference genes validated in roots, stems, and leaves of melons may be inappropriate for normalizing the target genes in melon fruits. The lack of validated reference genes specifically for melon fruits will certainly affect the accurate quantification of gene expression in melon fruits. Meanwhile, the availability of genome sequence (Garcia-Mas et al., 2012) and large-scale transcriptome data (Clepet et al., 2011; Portnoy et al., 2011; Saladié et al., 2015) will inevitably expedite the functional genomics research on melons, particularly on melon fruits. Therefore, selection of organ-specific reference genes with stable expression in melon fruits is vital to accurately explain the gene expression profiles during melon fruit development.

Fertilization and parthenocarpy are fruit set methods widely used for commercial melon production (Shin et al., 2007). In open field, fertilized fruits are often produced through natural pollination, while, under greenhouse conditions, parthenocarpic fruits are induced by the exogenous application of $\mathrm{N}$-(2chloro-4-pyridyl)- $N^{\prime}$-phenylurea (CPPU). CPPU is a synthetic cytokinin and can induce parthenocarpic fruit development in the absence of pollination and fertilization (Nagasawa et al., 2005). The two fruit set methods will trigger different patterns of gene expression and regulatory mechanisms during the subsequent fruit development, thereby providing a good opportunity for the selection of reference genes with intrinsically stable expression during melon fruit development. The sugar content and composition are major criteria used for assessment of melon fruit quality. Sucrose is the predominant sugar found in ripe melon fruits. Some previous studies have shown that soluble acid invertase (AI) and sucrose phosphate synthase (SPS) are the primary determinants closely associated with sucrose accumulation in melon fruits (Hubbard et al., 1989; Burger and Schaffer, 2007). Two AI genes (CmAIN1 and CmAIN2) and two SPS genes (CmSPS1 and CmSPS2) have been identified in the melon genome (Dai et al., 2011; Garcia-Mas et al., 2012). However, only CmAIN2 and CmSPS1 have been associated with sucrose accumulation in ripening melon fruits by deep sequencing analysis (Dai et al., 2011).

In this study, 20 candidate reference genes were selected, and their expression stability was evaluated at different development stages of fertilized and parthenocarpic melon fruits, with the aim of determining optimal reference genes for accurate quantification of target genes in melon fruits. Moreover, parallel analyses on the expression profiles of CmAIN2 and CmSPS1 normalized by the identified reference genes, and enzyme activities of AI and SPS, as well as sucrose accumulation during melon fruit ripening were performed to demonstrate the reliability of the identified reference genes.

\section{MATERIALS AND METHODS}

\section{Plant Materials and Treatments}

Melon (Cucumis melo L. var. inodorus cv. 'Elizabeth') plants were grown in a plastic greenhouse under commercial production conditions at the Huazhong Agricultural University (East Longitude $113^{\circ} 41^{\prime} 115^{\circ} 05^{\prime}$, North Latitude $29^{\circ} 58^{\prime} 31^{\circ} 22^{\prime}$ ). This cultivar is monoecious and without hermaphroditic flowers. To get fruits, two different fruit set methods were used, i.e., CPPU treatment and artificial pollination. For CPPU treatment, female flowers were covered with paper bags 1 day before anthesis to prevent natural pollination. These paper bags were removed from the female flowers on the day of anthesis, and the unpollinated ovaries were sprayed with CPPU (Shiteyou, China) at a concentration of $10 \mu \mathrm{M}$ in the morning. The female flowers were subsequently covered again after spraying till the onset of fruit development. Simultaneously, on other female flowers, artificial pollination was done by hand. Three biological replicates were adopted for each treatment. Fruits were harvested at 1, 3, 5, $7,10,15,20,25,28,30,32$, and 34 days after anthesis (DAA). Two fruits were randomly harvested at each sampling time from each biological replication and mixed together. Ovaries were sampled at 1 and 3 DAA. The mesocarp tissues in the center-equatorial portion of fruits were collected as samples after 3 DAA. All the samples were immediately frozen in liquid nitrogen and stored at $-80^{\circ} \mathrm{C}$ for the subsequent RNA extraction, enzyme assay, and sucrose measurement. 


\section{cDNA and DNA Preparation}

The qRT-PCR protocol (Nolan et al., 2006) and 11 golden rules (Udvardi et al., 2008) were used as guidelines in the experiments. Total RNA was extracted from the ovaries or mesocarp tissues with the TransZol (TransGen, China) according to its instruction. RNA quality and quantity were measured by a NanoDrop 2000 spectrophotometer (Thermo, China), and RNA integrity was confirmed in a $2 \%$ agarose-gel electrophoresis. A PrimeScript RT Reagent Kit with gDNA Eraser (Perfect Real Time; TaKaRa, China) was used to eliminate the genomic DNA (gDNA) in the RNA samples and synthesize the cDNA. gDNA was extracted from the mesocarp tissues with a Plant Genomic DNA Kit (Tiangen, China) and amplified using $2 \times$ PCR Reagent (Tiangen, China).

\section{Reference Genes Selection and Primer Design}

Fourteen genes were initially selected as the candidate reference genes. Table 1 lists the information on these candidate reference genes. For each gene, BLASTN was performed via the Melonomics database ${ }^{1}$ against the Melon transcripts CM_3.5. The Arabidopsis homologs were used as the query sequences. The sequence that best matched each Arabidopsis query was downloaded with its respective structure information. Primers that covered exon-exon junction or flanked an intron were designed using Primer3Plus ${ }^{2}$. The product size was set as $80-$ $150 \mathrm{bp}$. The specificity of the designed primers were manually verified and confirmed by running BLASTN against the Melon transcripts CM_3.5. The 2\% agarose-gel electrophoresis was further used to determine the PCR amplification specificity for each gene, with gDNA and cDNA as templets. The melon species name abbreviation "Cm" was adopted as a prefix to specify the orthologous melon genes. To make the results comparable, six best reference genes previously reported in melon, including ribosomal protein L2 (L2), actin*, CYP, ADP-ribosylation factor $1(A D P)$, ribosomal protein $L(R P L)$, and ubiquitin extension protein (UBI) that identified in stems, roots and leaves (Kong et al., 2014b; Sestili et al., 2014), were also selected. Their primer sequences were also used in the present study. The unigene accession numbers of actin* and CYP provided in the reference (Sestili et al., 2014) were used to retrieve the Melonomics database. The results showed that the two genes were annotated as actin 7 and CYP2 on melon genome, respectively. Their annotations and gene IDs on genome were used to replace the gene names and unigene accession numbers supplied in the previous reference (Sestili et al., 2014). The prefix of "Cm" was also added before names of the six reported reference genes.

\section{qRT-PCR Analysis}

Quantitative reverse transcription PCR reactions were performed on a QuantStudio 7 Flex Real-Time PCR System (The Applied Biosystems, America) using a total volume of $10 \mu \mathrm{L}$, which contained $0.2 \mu \mathrm{M}$ of each primer, $1 \times$ Top Green qPCR SuperMix

\footnotetext{
${ }^{1}$ https://melonomics.net/

${ }^{2}$ http://primer3plus.com/cgi-bin/dev/primer3plus.cgi
}

(TransGen, China), and100 ng of cDNA. The amplification conditions were $30 \mathrm{~s}$ at $94^{\circ} \mathrm{C}, 40$ cycles of $5 \mathrm{~s}$ at $95^{\circ} \mathrm{C}, 15 \mathrm{~s}$ at $58^{\circ} \mathrm{C}$ and $10 \mathrm{~s}$ at $72^{\circ} \mathrm{C}$, followed by a melting curve analysis by heating the PCR products from 65 to $95^{\circ} \mathrm{C}$. qRT-PCR reactions were performed in two technical replicates with a negative control without template. The PCR amplification efficiency for each gene was determined by analyzing fivefold serial dilutions of pooled cDNA in the concentrations of $800,160,32,6.4$, and

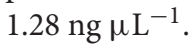

\section{Expression Stability Analysis}

The cycle threshold $(\mathrm{Ct})$ value was recorded for each qRTPCR reaction. The $\mathrm{R}$ statistical package ${ }^{3}$ was used to draw the boxplot to display the expression variation for each gene. The amplification efficiency (E) was calculated using the equation:

$$
\mathrm{E}(\%)=\left(10^{-1 / \text { slope }}-1\right) \times 100
$$

for each gene, in which the slope is the standard curve slope generated by the QuantStudio 7 Flex Real-Time PCR System based on the fivefold serial dilutions of pooled cDNA samples. The algorithms of geNorm (Vandesompele et al., 2002) and NormFinder (Andersen et al., 2004) were used to evaluate the expression variation. Prior to data entry, the raw $\mathrm{Ct}$ values were corrected by PCR efficiency and transformed into relative expression quantities using the equation $(1+\mathrm{E})^{\Delta \mathrm{Ct}}$, in which $\Delta \mathrm{Ct}$ is the difference of the lowest $\mathrm{Ct}$ value of the calibrator and the $\mathrm{Ct}$ value of the sample being tested.

\section{Determination of Sucrose Contents, Enzyme Activities of Al and SPS, and Expression Patterns of CmAIN2 and CmSPS1 during Fruit Ripening}

Pollinated fruits at $10,15,20,25,28$, and 32 DAA were used to determine the sucrose contents and enzyme activities. Extraction and measurement of sucrose were performed as previously described (Liu et al., 2012). The gas chromatograph Agilent 7890A (Agilent Technologies, USA) coupled with a HP5 capillary column $(30 \mathrm{~m} \times 0.32 \mathrm{~mm} \times 0.25 \mu \mathrm{m})$ and a CTC PAL autosampler (CTC Analytics, Switzerland) were used for sucrose detection and quantification. Extractions and activity measurements of AI and SPS were conducted as previously reported (Hubbard et al., 1989). CmAIN2 and CmSPS1 were selected to test the effectiveness of the identified reference genes. Primers of the two genes were designed according to the aforementioned methods and listed in Table 1. To demonstrate the transcriptional regulation of sucrose metabolism, an earlier sampling point at 7 DAA was added and used as control to analyze the expression levels of CmAIN2 and CmSPS1. qRT$\mathrm{PCR}$ reactions were performed according to the aforementioned methods. The $2^{-\Delta \Delta C t}$ method was used to calculate the relative expression level. The best reference genes identified by geNorm and NormFinder were used for normalization. Geometric means were calculated for the reference gene combinations and used for normalization. The widely used reference gene $\mathrm{CmCYP7}$ in

${ }^{3} \mathrm{http} / /$ www.r-project.org/ 
TABLE 1 | Information on the candidate reference genes, CmAIN2, and CmSPS1.

\begin{tabular}{|c|c|c|c|c|c|c|}
\hline Gene name & Description & Gene ID & $\begin{array}{l}\text { Forward primer } \\
\text { sequence }\left(5^{\prime}-3^{\prime}\right)\end{array}$ & $\begin{array}{l}\text { Reserve primer } \\
\text { sequence }\left(5^{\prime}-3^{\prime}\right)\end{array}$ & $\begin{array}{l}\text { Product } \\
\text { size (bp) }\end{array}$ & $E(\%)$ \\
\hline $\mathrm{CmACT}$ & $\beta$-Actin & MELO3C023264 & GAGCATCTAAACGGAGAGTTGG & GCCATCGTITATAGATACTTGAGGA & 104 & 98.9 \\
\hline CmCAC & $\begin{array}{l}\text { Clathrin adaptor complexs } \\
\text { mediun subunit family protein }\end{array}$ & MELO3C003397 & CCATTCTCATCCAAGCCTTC & TCAACAATATCCAAAAAGACCTCA & 125 & 100.6 \\
\hline CmCYP7 & Cyclophilin ROC7 & MELO3C025848 & TाTACCCTCGGCGATGGAAG & TGTGAACCATTGGTGTCTGGA & 134 & 99.4 \\
\hline CmEF1 $\alpha$ & Elongation factor $1-\alpha$ & MELO3C020441 & CTGCTTGCTCCTGCGTTAAA & CCACGATGTTGATATGAGTCTITC & 113 & 93.1 \\
\hline $\mathrm{CmGAPDH}$ & $\begin{array}{l}\text { Glyceraldehyde-3-phosphate } \\
\text { dehydrogenase }\end{array}$ & MELO3C019633 & CATGGTGTIITCAACGGAACCA & CCCATGGGATATCTGCAGGG & 110 & 103.8 \\
\hline $\mathrm{CmPP} 2 \mathrm{~A}$ & $\begin{array}{l}\text { Protein phosphatase } 2 \mathrm{~A} \\
\text { regulatory subunit } \mathrm{A}\end{array}$ & MELO3C026508 & GGCAGATAACTCAAGTITATGGA & $\begin{array}{l}\text { GCTGTAAGAGGTAAATAATCA } \\
\text { AAGAGG }\end{array}$ & 109 & 94.8 \\
\hline CMRAN & RAN GTPase & MELO3C026633 & AAGACATCTCACAGGGGAGTT & AGCAGTGTCCCAGCAGTAAA & 118 & 96.6 \\
\hline $\mathrm{CmRP2}$ & RNA polymerases II & MELO3C008589 & GCGCTGGATACCAAAGGAAT & TGCGTGATCTITACCAATGC & 101 & 105.5 \\
\hline CmRPS15 & Cytosolic ribosomal protein S15 & MELO3C006471 & GAAGCTGCGTAAAGCGAAAC & GGTCTITCCATTGTAAACTCCAA & 132 & 108.8 \\
\hline CmSAND & SAND family protein & MELO3C004874 & TATCGTGGAGGAAAAGGAAGAAGC & CTCGTCCCCGTACCTGGAAT & 80 & 105.1 \\
\hline CmTBP2 & TATA-box binding protein & MELO3C015563 & GGAAACATATACGGCTITGAGA & TTCGAAACCAAAAATCATTGC & 81 & 107.8 \\
\hline CmTIP41 & TIP41-like family protein & MELO3C018500 & GGTAATCTTGTATGAGGATGAGCTG & CATCAACTCTAAGCCAGAAACG & 118 & 107.4 \\
\hline CmTUA5 & Tubulin alpha-5 & MELO3C026613 & AGGACTGGGACATACCGACA & CGGCTAATITCGCACTCGG & 145 & 99.1 \\
\hline CmYLS8 & Yellow-leaf-specific gene 8 & MELO3C020882 & GTGGTCATTCGTITGGTCA & CAGCAAAGTTCTTAATCGTCTCT & 94 & 108.0 \\
\hline CmACT7 & Actin* & MELO3C008032 & CCCTGGTATTGCAGACAGGA & ACATCTGCTGGAAGGTGCTT & 149 & 105.2 \\
\hline CmCYP2 & Cyclophilin & MELO3C013375 & CACACCGGACCTGGTATTCT & CATCCATACCCTCGACGACT & 139 & 107.4 \\
\hline $\mathrm{CmL2}$ & Ribosomal protein L2 & MELO3C000111 & AAACTTCTACCCCGAGCACA & TATGACCTCCCCCTCTATGC & 150 & 109.3 \\
\hline$C m A D P$ & ADP ribosylation factor 1 & MELO3C023630 & ATATTGCCAACAAGGCGTAGA & TGCCCGTAAACAAGGGATAAA & 93 & 98.2 \\
\hline CmRPL & Ribosomal protein $\mathrm{L}$ & MELO3C023039 & CGACAATACTGGAGCCAAGAA & CATCACCATATCTCCCACACAA & 100 & 104.6 \\
\hline$C m \cup B I$ & Ubiquitin extension protein & MELO3C016083 & AAGTGTGGACACAGCAACCA & AAGCCAAATGGCTCTAAGCA & 132 & 94.5 \\
\hline CmAIN2 & Acid invertase 2 & MELO3C005363 & AATGACGTGCTCCTCGTACC & ТTССАСТTСАAАСТССGСCA & 90 & \\
\hline CmSPS1 & Sucrose phosphate synthase 1 & MELO3C010300 & GACACTTCAGTCCCACTCGG & TCTAGTATTCСTCTCCTGCGGA & 122 & \\
\hline
\end{tabular}

melon fruits and the least stable reference gene identified in this study were additionally used for normalization. Three biological and two technical replicates were adopted for the aforementioned measurements at each sampling point.

\section{RESULTS}

\section{PCR-Amplification Characteristics of the Candidate Reference Genes}

A total of 20 genes were selected as the candidate reference genes in this study, which included the six reference genes that previously identified in melon roots, leaves, and stems (Kong et al., 2014b; Sestili et al., 2014). Information on these 20 candidate reference genes is listed in Table 1. Primer sequences of the six reference genes previously identified in melon (Kong et al., 2014b; Sestili et al., 2014) were also used in this study. While, primer sequences of the other 14 candidate reference genes were designed.

To improve the specificity of qRT-PCR analysis, one primer of each gene was initially designed to match an exon-exon junction. However, a suitable primer binding site was difficult to find in the exon-exon junction regions of some genes. Primers of the said genes were then located on the exons separated by an intron. Amplification specificity of the 20 genes was determined by a $2 \%$ agarose-gel electrophoresis with cDNA and genomic DNA (gDNA) as templates, respectively
(Figure 1). The results showed that only the expected products were amplified with cDNA templates and no products were amplified with gDNA templates for $C m A C T, C m C A C$, CmPP2A, CmRP2, CmRPS15, CmSAND, CmTBP2, and CmTIP41. Meanwhile, CmCYP7, CmEF1 $\alpha$, CmGAPDH, CmRAN, CmYLS8, and CmTUA5 amplified specific products from both cDNA and gDNA templates. However, the amplicons from gDNA templates containing an intron are larger than those from cDNA templates, demonstrating the success of primer design and the absence of gDNA contaminations in the cDNA samples. Among the six previously identified reference genes in melon, CmACT7, $C m C Y P 2, C m L 2$, and $C m R P L$ amplified the same products on both gDNA and cDNA templates, whereas, $C m A D P$ had no products and $C m U B I$ generated a larger product on gDNA templates, respectively. Meanwhile, a single peak in the melting curve analysis further supported the specific amplification of each gene. Amplification efficiencies of the 20 genes varied from $93.1 \%$ $(C m E F 1 \alpha)$ to $109.3 \%$ (CmL2). Table 1 summarizes the primer sequences and amplification characteristics of the 20 genes.

\section{Expression Stability of the Candidate Reference Genes}

Expression variations of the 20 genes were examined during the developments of pollinated and CPPU treated fruits and represented as boxplot in Figure 2. The mean Ct values for the 20 genes across the 24 samples ranged from 19.0 (CmCYP2) to 29.4 

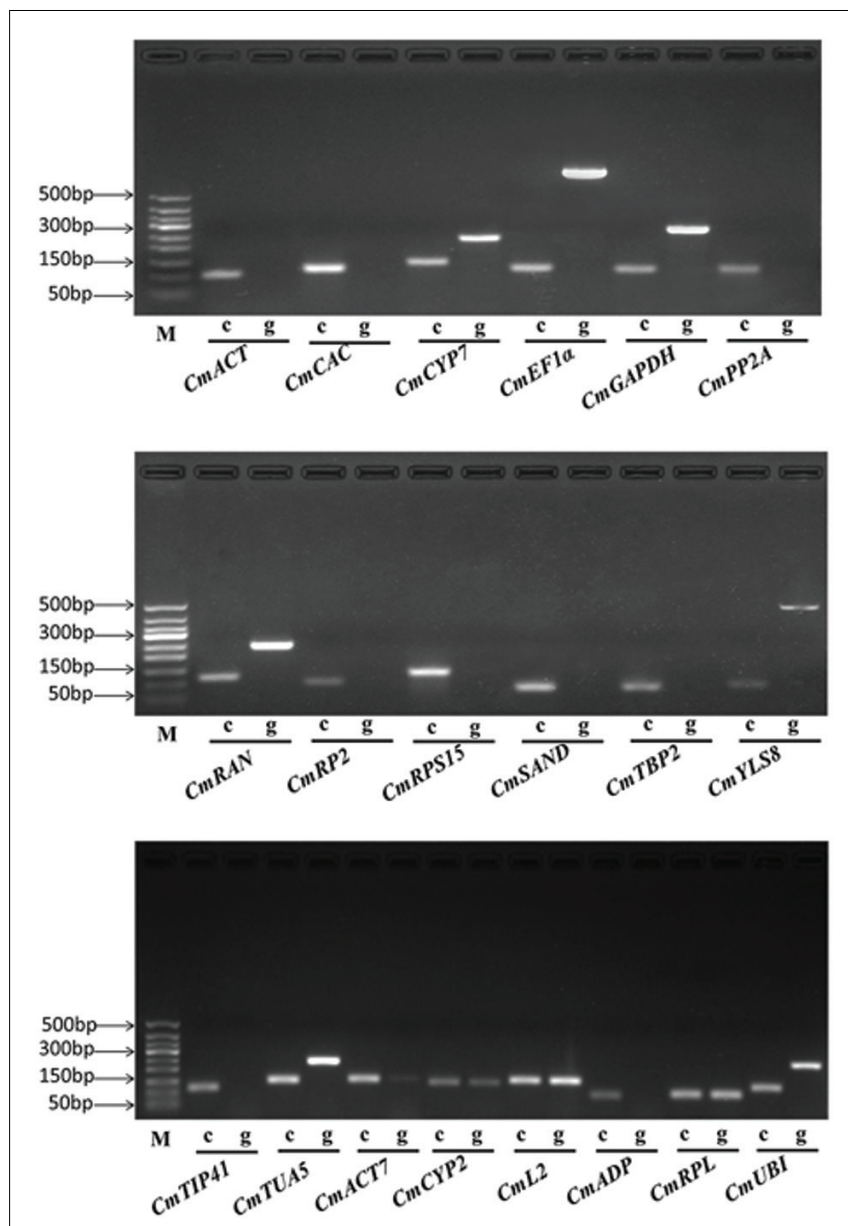

FIGURE 1 | Polymerase chain reaction (PCR) amplification products of 20 reference genes in $\mathbf{2} \%$ agarose gel. ' $c$ ' indicates the cDNA template, ' $g$ ' indicates the gDNA template, ' $M$ ' indicates the DNA ladder marker.
(CmSAND). CmADP exhibited the least expression variation, whereas CmRP2, CmRAN, CmGAPDH, and CmEF1a showed the highest expression variations. Given the presences of nonbiological variations in the experiments, gene expression stability cannot be accurately estimated by direct comparison of the raw $\mathrm{Ct}$ values. Therefore, geNorm and NormFinder were used to assess the expression stability.

GeNorm calculates the expression stability value (M) for each gene and considers that gene with lower $\mathrm{M}$ value has higher expression stability. Taking into account the effects of fruit set methods and developmental phases, geNorm ranked CmRPL and CmRPS15 as the pair of best reference genes, and CmUBI as the least stable gene (Figure 3A). Furthermore, geNorm determines the optimum number of reference genes required for reliable normalization by calculating the pairwise variation values $(\mathrm{V})$. When the pairwise variation $\left(V_{n / n+1}\right)$ is less than 0.15 , it is recommended that no additional genes are required for the normalization. Pairwise variation analysis demonstrated that at least five reference genes were required for more reliable normalization, namely, CmRPL, CmRPS15, CmTIP41, CmCYP7, and $C m A D P$ (Figure 3B).

NormFinder uses a model-based approach and considers variations across groups to calculate the expression stability for each gene. The gene with lower stability value is top ranked. The samples were divided into fertilized and parthenocarpic groups according to the fruit set methods. The plots of inter- and intragroup variations with respect to fruit set methods for each gene showed that CmTIP41 had the lowest intergroup variation, whereas CmACT7 exhibited the lowest intragroup variation (Figure 4). CmRPS15 was determined as the best reference gene with the lowest stability value of 0.268 . Meanwhile, NormFinder identified CmRAN and CmTBP2 as the combination of two best reference genes, with minimal combined inter- and intragroup variations. $C m E F 1 \alpha$ was ranked as the least stable reference gene (Figure 4).

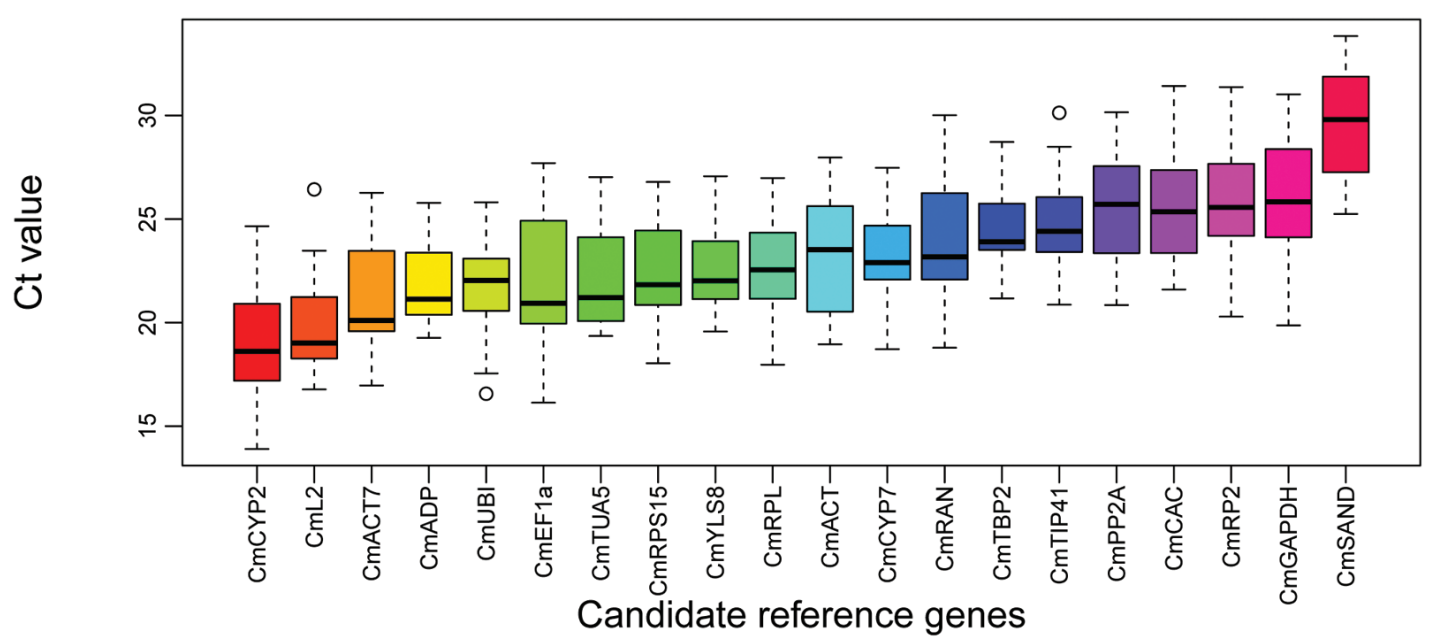

FIGURE 2 | Boxplot analysis on expression profiles of the candidate reference genes across all 24 samples. The line across the box represents the median. The boxes represent the $25 / 75$ percentiles. The whiskers show the maximum and minimum values. The circles indicate the outliers. 
A

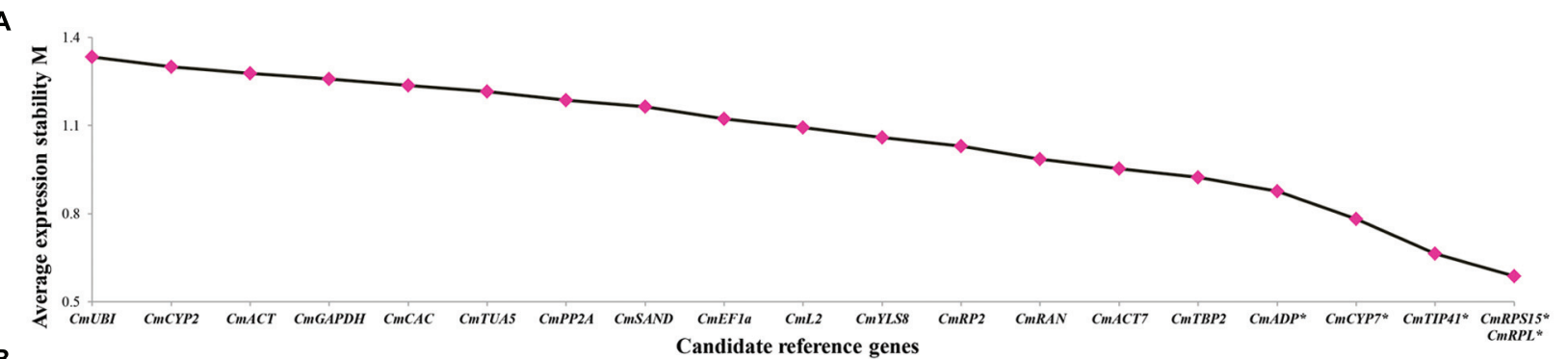

B $<==$ Least stable gene Most stable gene $==>$

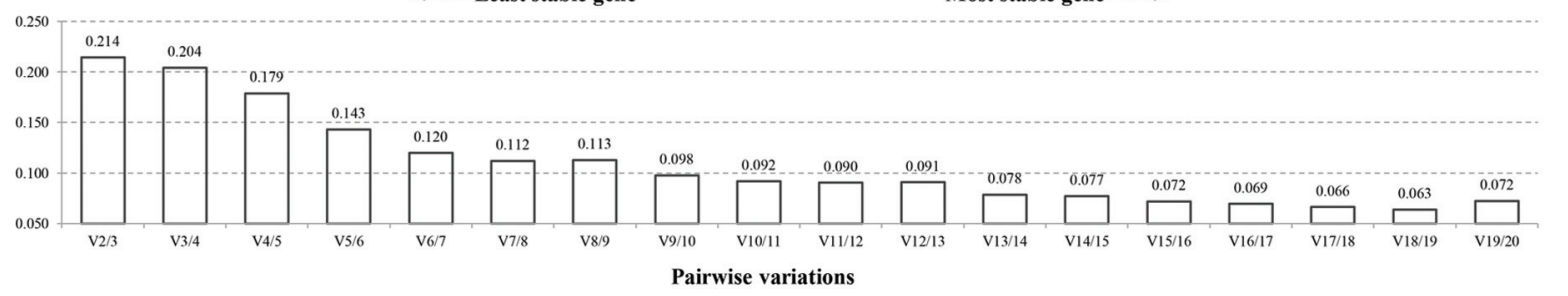

FIGURE 3 | geNorm analysis of the candidate reference genes. Genes are ordered by descending M values (A), and the minimum number of reference genes required for reliable normalization is demonstrated by pair-wise variation analysis (B). The asterisks indicate the reference genes needed for more reliable normalization when the cutoff value 0.15 was adopted $(\mathbf{A})$.

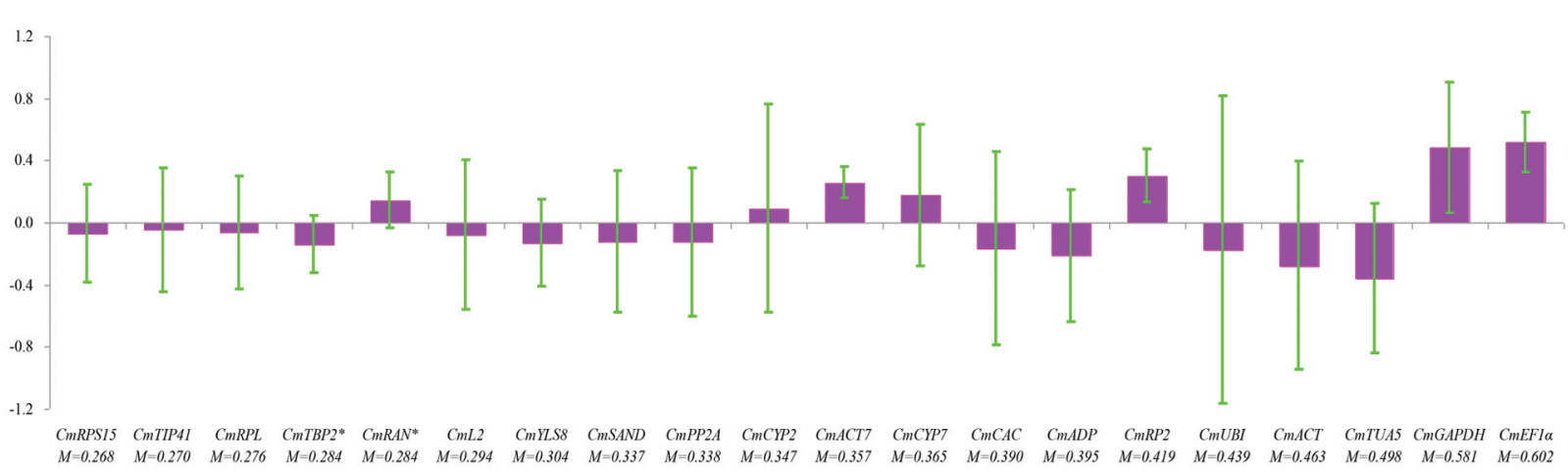

FIGURE 4 | NormFinder analysis of the candidate reference genes. The samples were divided into two subgroups according to method of fruit set. The histogram displays the intergroup variation. The error bars represent the intragroup variation. M represents the stability value. Asterisks indicate the combination of two best genes.

\section{Validation of the Identified Reference Genes}

Parallel changes of sucrose contents, activities of AI and SPS, and expression patterns of CMAIN2 and CmSPS1 were analyzed during fruit ripening (Figure 5). The AI activity was highest at $10 \mathrm{DAA}$ and then decreased sharply with fruit ripening (Figure 5A). The SPS activity gradually increased from 10 DAA and reached the highest level at $25 \mathrm{DAA}$, then decreased and kept stable till fruit matured (Figure 5B). The sucrose contents were very low before 20 DAA and then gradually increased with fruit ripening (Figure 5C). The best genes that determined by geNorm and NormFinder were used to normalize the expression levels of CmAIN2 and CmSPS1, respectively. These genes included the single gene CmRPS15, combination of CmRPS15 and CmRPL, combination of CmRAN and CmTBP2, and multiple genes of CmRPL, CmRPS15, CmTIP41, CmCYP7, and $C m A D P$. Meanwhile, $C m C Y P 7$ and the least stable gene $C m U B I$ determined by geNorm were also used for normalization. The relative expression levels of CmAIN2 gradually decreased after 7 DAA, and were nearly undetectable after 20 DAA when the best reference genes were used for normalization (Figure 5A), which were in agreement with the changing patterns of AI activities. However, when CmUBI or CmCYP7 was used for normalization, the expression levels of CmAIN2 increased from 7 to $10 \mathrm{DAA}$ and then decreased as fruit ripening. CmSPS1 was upregulated from 7 to $15 \mathrm{DAA}$, then gradually downregulated as fruit matured, no matter the stable or less stable genes were used for normalization (Figure 5B). However, compared with the expression levels normalized by the best genes, the expression levels of CmSPS1 were obviously overestimated from 15 to 28 DAA when the less stable gene CmUBI or CmCYP7 was used for normalization. 

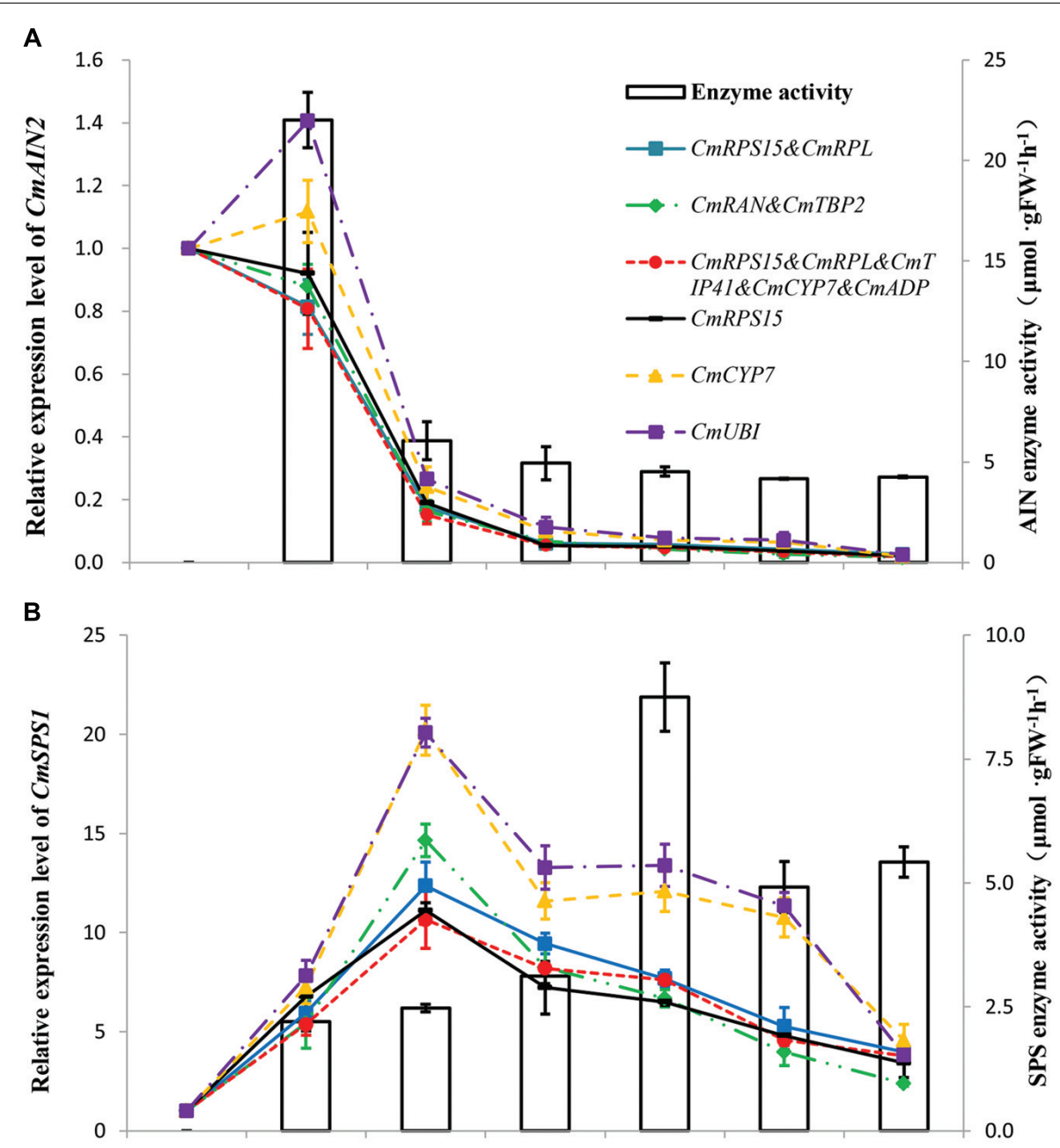

C

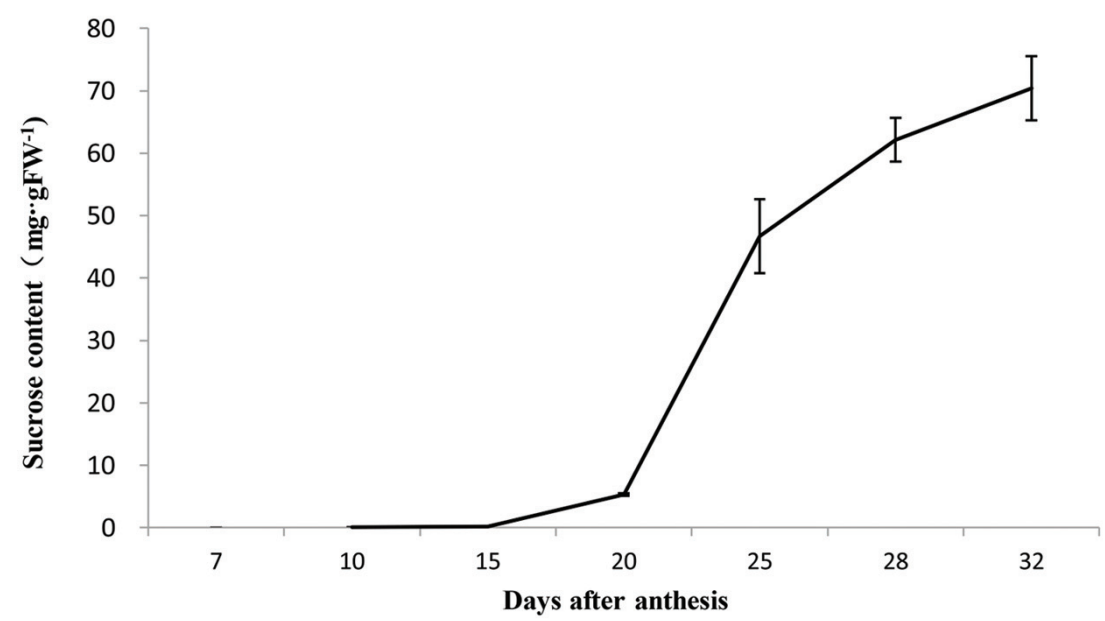

FIGURE 5 | Validation of the identified reference genes. Al enzyme activity and CmAIN2 expression profile (A), SPS enzyme activity and CmSPS1 expression profile (B), as well as sucrose content (C) were measured during melon fruit development. The relative expression levels of CmAIN2 and CmSPS1 were normalized by the single best reference gene (CmRPS15 determined by NormFinder), the pair of best reference genes (CmRPS15 and CmRPL identified by geNorm, and CmRAN and CmTBP2 identified by NormFinder), the multiple reference genes (CmRPL, CmRPS15, CmTIP41, CmCYP7, and CmADP identified by geNorm), the least stable reference gene (CmUB/ determined by geNorm), and the previously used reference gene in melon fruits (CmCYP7), respectively. Transcript abundance of 7 DAA was used as control. The results are depicted as the mean \pm SE $(n=6)$. 


\section{DISCUSSION}

Expression analysis is a crucial step to gain insight into the biological functions of genes. However, systematic validation of reference genes has not been performed for qRT-PCR-based gene expression studies in melon fruits to date. The use of nonvalidated reference genes for normalization does not meet the prerequisites of qRT-PCR and thus may introduces bias into the final results (Gutierrez et al., 2008; Bustin et al., 2009). In this study, 20 candidate reference genes were systematically examined for their potential use as reference genes in melon fruits.

To improve the specificity of qRT-PCR analysis, gene-specific primers locating at the exon-exon junctions or two adjoining exons were designed for the 14 candidate reference genes in this study. The similar strategy was also used in Arabidopsis (Czechowski et al., 2005), strawberry (Amil-Ruiz et al., 2013), and watermelon (Kong et al., 2014a, 2015). The primer pairs developed in the present study can detect or control the potential gDNA contaminations in cDNA samples, and thus can be used in the related studies.

NormFinder and geNorm are the widely used algorithms to identify suitable reference genes. Both geNorm and NormFinder demonstrated that the previously used ACT, CYP7, and GAPDH were not the optimal reference genes in melon fruits. CmACT and $C m G A P D H$ were ranked the second to fourth from the last by geNorm and NormFinder. Only CmCYP7 was in the multiple reference genes determined by geNorm. Moreover, the unstable expression of these genes has been demonstrated in the fruits of other crops, such as papaya (Zhu et al., 2012) and blueberry (Die and Rowland, 2013). As far as the six best reference genes that identified in the melon roots, leaves, and stems are concerned (Kong et al., 2014b; Sestili et al., 2014), only CmRPL was in the pair of best genes and $C m A D P$ was in the multiple genes that determined by geNorm in melon fruits, indicating the organspecific characteristics of reference genes and importance of identifying appropriate reference genes specifically for melon fruits.

NormFinder identified CmRPS15 as the single best reference gene, and geNorm identified CmRPS15 and CmRPL as the pair of best reference genes in melon fruits. RPS15, initially called as rig, has been proven to be a housekeeping gene in human (Shiga et al., 1990; Kitagawa et al., 1991) and was identified as the suitable reference gene in many cases in mammals (Bionaz and Loor, 2007; Kumar et al., 2012). The housekeeping feature of PRS15 also supported the suitability of CmRPS15 as reference gene in melon fruits. Meanwhile, RPS2, the other member of $R P S$ gene family was also identified as the suitable reference gene in banana fruits (Chen et al., 2011). The ribosomal protein related genes $C m P R L$ and $C m L 2$ were ranked among the suitable reference genes in melon roots, stems, and leaves in the previous studies (Kong et al., 2014b; Sestili et al., 2014). In this study, $C m R P L$ was also identified as one of the suitable reference genes in melon fruits, suggesting that ribosomal protein related genes were potentially the widely applicable reference genes in melon. NormFinder identified CmTBP2 and CmRAN as the combination of two best reference genes, Similarly, TBP2 was ranked among the suitable reference genes in papaya fruits (Zhu et al., 2012).
However, RAN was not reported as the suitable reference genes in the fruits of other crops in the previous studies, indicating the importance of identifying suitable reference genes for specific species.

Acid invertase and SPS were considered the determinants of sucrose concentration in developing melon fruits (Hubbard et al., 1989; Burger and Schaffer, 2007). Thus, parallel analyses of the activities of AI and SPS, and expression patterns of their respective encoding genes, as well as sucrose accumulation during melon fruit ripening, can provide reliable measurement to test the suitability of the identified reference genes. Increased sucrose contents accompanied by the decreased AI activities were observed as fruit matured. Meanwhile, SPS activities also tended to be increased during fruit ripening, although its highest level occurred at 25 DAA. These results were in general agreement with the previous reports on sucrose metabolism in melon fruits (Hubbard et al., 1989; Burger and Schaffer, 2007; Dai et al., 2011). The expression patterns of CmAIN2 were nearly identical regardless the best single, pair of, or multiple reference genes were used for normalization. They exhibited positive correlation with the developmental changes of AI activity and negative correlation with the sucrose accumulation during fruit development. Similar results were also observed in ripening melon fruits by deep sequencing analysis (Dai et al., 2011). No obvious correlation was observed between expression patterns of CmSPS1 and SPS activities or sucrose contents during fruit development regardless the stable or unstable reference genes were used for normalization, suggesting that CmSPS1 was possibly not the major gene regulating sucrose metabolism on transcriptional level. CmUBI was ranked as the least stable gene by geNorm. Although CmCYP7 was among the multiple reference genes determined by the pairwise variation analysis of geNorm, its expression stability was less than that of the identified best single or pairs of genes. Compared with the best single reference gene or gene combinations, the expression levels of CmAIN2 and CmSPS1 were overestimated at some sampling points when the less stable gene $\mathrm{CmUBI}$ or $\mathrm{CmCYP7}$ was used for normalization, which highlighted the importance of selecting the systematically validated reference genes in qRT-PCR analysis. Although different best single reference gene or gene combinations were identified by geNorm and NormFinder, the expression patterns of CmAIN2 or CmSPS1 were nearly identical when these identified genes were used for normalization, and in general agreement with the previous reports or the changing patterns of their respective encoding protein activities, demonstrating the suitability of the identified reference genes in melon fruits.

\section{CONCLUSION}

The suitability of 20 genes for their potential use as reference genes in melon fruits was assessed in this study. For more reliable normalization, the multiple genes including CmRPL, CmRPS15, $C m T I P 41, C m C Y P 7$, and $C m A D P$ were required in melon fruits. However, taking account the cost and similar normalization results as the multiple reference genes, CmRPS15 alone or 
together with $C m R P L$, or combination of CmRAN and CmTBP2 were the preferred reference gene or reference gene combinations that can be used to replace the non-validated reference genes that previously used in melon fruits. This study offers a reference gene selection guideline specifically for melon fruits and provides valuable information for further studies on the transcriptional regulation of sucrose metabolism during melon fruit ripening.

\section{AUTHOR CONTRIBUTIONS}

QK and ZB conceived and designed the experiments. LG, LC, $\mathrm{YL}$, and HS performed the experiments. QK and LG analyzed

\section{REFERENCES}

Amil-Ruiz, F., Garrido-Gala, J., Blanco-Portales, R., Folta, K. M., Munoz-Blanco, J. and Caballero, J. L. (2013). Identification and validation of reference genes for transcript normalization in strawberry (Fragaria $\mathrm{x}$ ananassa) defense responses. PLoS ONE 8:e70603. doi: 10.1371/journal.pone.0070603

Andersen, C. L., Jensen, J. L., and Orntoft, T. F. (2004). Normalization of real-time quantitative reverse transcription-PCR data: a model-based variance estimation approach to identify genes suited for normalization, applied to bladder and colon cancer data sets. Cancer Res. 64, 5245-5250. doi: 10.1158/0008-5472.can04-0496

Bionaz, M., and Loor, J. J. (2007). Identification of reference genes for quantitative real-time PCR in the bovine mammary gland during the lactation cycle. Physiol. Genomics 29, 312-319. doi: 10.1152/physiolgenomics.00223.2006

Burger, Y., and Schaffer, A. A. (2007). The contribution of sucrose metabolism enzymes to sucrose accumulation in Cucumis melo. J. Am. Soc. Hortic. Sci. 132, $704-712$.

Bustin, S. A., Benes, V., Garson, J. A., Hellemans, J., Huggett, J., Kubista, M., et al. (2009). The MIQE guidelines: minimum information for publication of quantitative real-time PCR experiments. Clin. Chem. 55, 611-622. doi: 10.1373/clinchem.2008.112797

Chen, L., Zhong, H. -Y., Kuang, J. -F., Li, J. -G., Lu, W. -J., and Chen, J. -Y. (2011). Validation of reference genes for RT-qPCR studies of gene expression in banana fruit under different experimental conditions. Planta 234, 377-390. doi: 10.1007/s00425-011-1410-3

Clepet, C., Joobeur, T., Zheng, Y., Jublot, D., Huang, M., Truniger, V., et al. (2011). Analysis of expressed sequence tags generated from full-length enriched cDNA libraries of melon. BMC Genomics 12:252. doi: 10.1186/1471-2164-12-252

Czechowski, T., Stitt, M., Altmann, T., Udvardi, M. K., and Scheible, W. R. (2005). Genome-wide identification and testing of superior reference genes for transcript normalization in Arabidopsis. Plant Physiol. 139, 5-17. doi: 10.1104/pp.105.063743

Dai, N., Cohen, S., Portnoy, V., Tzuri, G., Harel-Beja, R., Pompan-Lotan, M., et al. (2011). Metabolism of soluble sugars in developing melon fruit: a global transcriptional view of the metabolic transition to sucrose accumulation. Plant Mol. Biol. 76, 1-18. doi: 10.1007/s11103-011-9757-1

Dheda, K., Huggett, J. F., Chang, J. S., Kim, L. U., Bustin, S. A., Johnson, M. A., et al. (2005). The implications of using an inappropriate reference gene for real-time reverse transcription PCR data normalization. Anal. Biochem. 344, 141-143. doi: 10.1016/j.ab.2005.05.022

Díaz, A., Zarouri, B., Fergany, M., Eduardo, I., Álvarez, J. M., Picó, B., et al. (2014). Mapping and introgression of QTL involved in fruit shape transgressive segregation into 'Piel de Sapo' Melon (Cucucumis melo L.). PLoS ONE 9:e104188. doi: 10.1371/journal.pone.0104188

Die, J. V., and Rowland, L. J. (2013). Superior cross-species reference genes: a blueberry case study. PLoS ONE 8:e73354. doi: 10.1371/journal.pone.0073354

Ezura, H., and Owino, W. O. (2008). Melon, an alternative model plant for elucidating fruit ripening. Plant Sci. 175, 121-129. doi: 10.1016/j.plantsci.2008.02.004

Galpaz, N., Burger, Y., Lavee, T., Tzuri, G., Sherman, A., Melamed, T., et al. (2013). Genetic and chemical characterization of an EMS induced mutation the data. YH contributed reagents/materials. QK wrote the manuscript.

\section{ACKNOWLEDGMENTS}

This work was funded by the National Natural Science Foundation of China (31471894), the National Science and Technology Pillar Program during the 12th 5-years Plan Period (2012BAD02B03-16), the earmarked fund for Modern Agroindustry Technology Research System (CARS-26-16), and the Special Fund for Agro-scientific Research in the Public Interest (201203080-2).

in Cucumis melo CRTISO gene. Arch. Biochem. Biophys. 539, 117-125. doi: 10.1016/j.abb.2013.08.006

Gao, F., Niu, Y. -D., Hao, J. -F., Bade, R., Zhang, L. -Q., and Hasi, A. (2013). Identification of differentially expressed genes during ethylene climacteric of melon fruit by suppression subtractive hybridization. J. Integr. Agric. 12, 14311440. doi: 10.1016/s2095-3119(13)60551-1

Garcia-Mas, J., Benjak, A., Sanseverino, W., Bourgeois, M., Mir, G., Gonzalez, V. M., et al. (2012). The genome of melon (Cucumis melo L.). Proc. Natl. Acad. Sci. U.S.A. 109, 11872-11877. doi: 10.1073/pnas.1205415109

Gonda, I., Lev, S., Bar, E., Sikron, N., Portnoy, V., Davidovich-Rikanati, R., et al. (2013). Catabolism of L-methionine in the formation of sulfur and other volatiles in melon (Cucumis melo L.) fruit. Plant J. 74, 458-472. doi: $10.1111 /$ tpj.12149

Gonzalez-Ibeas, D., Blanca, J., Roig, C., Gonzalez-To, M., Pico, B., Truniger, V., et al. (2007). MELOGEN: an EST database for melon functional genomics. BMC Genomics 8:306. doi: 10.1186/1471-2164-8-306

Guénin, S., Mauriat, M., Pelloux, J., Van Wuytswinkel, O., Bellini, C., and Gutierrez, L. (2009). Normalization of qRT-PCR data: the necessity of adopting a systematic, experimental conditions-specific, validation of references. J. Exp. Bot. 60, 487-493. doi: 10.1093/jxb/ern305

Gutierrez, L., Mauriat, M., Guenin, S., Pelloux, J., Lefebvre, J. -F., Louvet, R., et al. (2008). The lack of a systematic validation of reference genes: a serious pitfall undervalued in reverse transcription-polymerase chain reaction (RTPCR) analysis in plants. Plant Biotechnol. J. 6, 609-618. doi: 10.1111/j.14677652.2008.00346.x

Hubbard, N. L., Huber, S. C., and Pharr, D. M. (1989). Sucrose phosphate synthase and acid invertase as determinants of sucrose concentration in developing muskmelon (Cucumis melo L.) fruits. Plant Physiol. 91, 1527-1534. doi: 10.1104/pp.91.4.1527

Huggett, J., Dheda, K., Bustin, S., and Zumla, A. (2005). Real-time RT-PCR normalisation; strategies and considerations. Genes Immun. 6, 279-284. doi: 10.1038/sj.gene.6364190

Kitagawa, M., Takasawa, S., Kikuchi, N., Itoh, T., Teraoka, H., Yamamoto, H., et al. (1991). rig encodes ribosomal protein S15 The primary structure of mammalian ribosomal protein S15. FEBS Lett. 283, 210-214. doi: 10.1016/00145793(91)80590-Y

Kong, Q., Yuan, J., Gao, L., Zhao, L., Cheng, F., Huang, Y., et al. (2015). Evaluation of appropriate reference genes for gene expression normalization during watermelon fruit development. PLoS ONE 10:e0130865. doi: 10.1371/journal.pone.0130865

Kong, Q., Yuan, J., Gao, L., Zhao, S., Jiang, W., Huang, Y., et al. (2014a). Identification of suitable reference genes for gene expression normalization in qrt-pcr analysis in watermelon. PLoS ONE 9:e90612. doi: 10.1371/journal.pone.0090612

Kong, Q., Yuan, J., Niu, P., Xie, J., Jiang, W., Huang, Y., et al. (2014b). Screening suitable reference genes for normalization in reverse transcription quantitative Real-Time PCR analysis in melon. PLoS ONE 9:e87197. doi: 10.1371/journal.pone.0087197

Kumar, P., Yadav, P., Verma, A., Singh, D., De, S., and Datta, T. K. (2012). Identification of stable reference genes for gene expression studies using quantitative real time PCR in buffalo oocytes and embryos. 
Reprod. Domest. Anim. 47, e88-e91. doi: 10.1111/j.1439-0531.2012. 01998.x

Leida, C., Moser, C., Esteras, C., Sulpice, R., Lunn, J. E., de Langen, F., et al. (2015). Variability of candidate genes, genetic structure and association with sugar accumulation and climacteric behavior in a broad germplasm collection of melon (Cucumis melo L.). BMC Genet. 16:28. doi: 10.1186/s12863-015-0183-2

Liu, C., Zhang, H., Dai, Z., Liu, X., Liu, Y., Deng, X., et al. (2012). Volatile chemical and carotenoid profiles in watermelons [Citrullus vulgaris (Thunb.) schrad (Cucurbitaceae)] with different flesh colors. Food Sci. Biotechnol. 21, 531-541. doi: 10.1007/s10068-012-0068-3

Moreno, E., Obando, J. M., Dos-Santos, N., Fernández-Trujillo, J. P., Monforte, A. J., and Garcia-Mas, J. (2008). Candidate genes and QTLs for fruit ripening and softening in melon. Theor. Appl. Genet. 116, 589-602. doi: 10.1007/s00122007-0694-y

Nagasawa, M., Mori, H., Shiratake, K., and Yamaki, S. (2005). Isolation of cDNAs for genes expressed after/during fertilization and fruit set of melon (Cucumis melo L.). J. Jpn. Soc. Horticult. Sci. 74, 23-30. doi: 10.2503/jjshs.74.23

Nolan, T., Hands, R. E., and Bustin, S. A. (2006). Quantification of mRNA using real-time RT-PCR. Nat. Protoc. 1, 1559-1582. doi: 10.1038/nprot.2006.236

Nunez-Palenius, H. G., Gomez-Lim, M., Ochoa-Alejo, N., Grumet, R., Lester, G., and Cantliffe, D. J. (2008). Melon fruits: genetic diversity, physiology, and biotechnology features. Crit. Rev. Biotechnol. 28, 13-55. doi: 10.1080/07388550801891111

Portnoy, V., Diber, A., Pollock, S., Karchi, H., Lev, S., Tzuri, G., et al. (2011). Use of Non-Normalized, Non-amplified cDNA for 454-based RNA sequencing of fleshy melon fruit. Plant Genome 4, 36-46. doi: 10.3835/plantgenome2010.11.0026

Qin, X., Coku, A., Inoue, K., and Tian, L. (2011). Expression, subcellular localization, and cis-regulatory structure of duplicated phytoene synthase genes in melon (Cucumis melo L.). Planta 234, 737-748. doi: 10.1007/s00425-0111442-8

Saladié, M., Cañizares, J., Phillips, M. A., Rodriguez-Concepcion, M., Larrigaudière, C., Gibon, Y., et al. (2015). Comparative transcriptional profiling analysis of developing melon (Cucumis melo L.) fruit from climacteric and non-climacteric varieties. BMC Genomics 16:440. doi: 10.1186/s12864-015-1649-3

Sestili, S., Sebastiani, M. S., Belisario, A., and Ficcadenti, N. (2014). Reference gene selection for gene expression analysis in melon infected by Fusarium oxysporum f. sp. melonis. J. Plant Biochem. Biotechnol. 23, 238-248. doi: 10.1007/s13562013-0207-9
Shan, W., Zhao, C., Fan, J., Cong, H., Liang, S., and Yu, X. (2012). Antisense suppression of alcohol acetyltransferase gene in ripening melon fruit alters volatile composition. Sci. Horticult. 139, 96-101. doi: 10.1016/j.scienta.2012.03.010

Shiga, K., Yamamoto, H., and Okamoto, H. (1990). Isolation and characterization of the human homologue of rig and its pseudogenes: the functional gene has features characteristic of housekeeping genes. Proc. Natl. Acad. Sci. U.S.A. 87, 3594-3598. doi: 10.1073/pnas.87.9.3594

Shin, Y. S., Park, S. D., and Kim, J. H. (2007). Influence of pollination methods on fruit development and sugar contents of oriental melon (Cucumis melo L. cv. Sagyejeol-Ggul). Sci. Horticult. 112, 388-392. doi: 10.1016/j.scienta.2007. 01.025

Sun, Y., Chen, P., Duan, C., Tao, P., Wang, Y., Ji, K., et al. (2013). Transcriptional regulation of genes encoding key enzymes of abscisic acid metabolism during melon (Cucumis melo L.) fruit development and ripening. J. Plant Growth Regul. 32, 233-244. doi: 10.1007/s00344-012-9293-5

Udvardi, M. K., Czechowski, T., and Scheible, W. (2008). Eleven golden rules of quantitative RT-PCR. Plant Cell 20, 1736-1737. doi: 10.1105/tpc.108.061143

Vandesompele, J., De Preter, K., Pattyn, F., Poppe, B., Van Roy, N., De Paepe, A., et al. (2002). Accurate normalization of real-time quantitative RT-PCR data by geometric averaging of multiple internal control genes. Genome Biol. 3:RESEARCH0034. doi: 10.1186/gb-2002-3-7-research0034

Vegas, J., Garcia-Mas, J., and Monforte, A. J. (2013). Interaction between QTLs induces an advance in ethylene biosynthesis during melon fruit ripening. Theor. Appl. Genet. 126, 1531-1544. doi: 10.1007/s00122-013-2071-3

Zhu, X., Li, X., Chen, W., Chen, J., Lu, W., Chen, L., et al. (2012). Evaluation of new reference genes in papaya for accurate transcript normalization under different experimental conditions. PLoS ONE 7:e44405. doi: 10.1371/journal.pone.0044405

Conflict of Interest Statement: The authors declare that the research was conducted in the absence of any commercial or financial relationships that could be construed as a potential conflict of interest.

Copyright (C) 2016 Kong, Gao, Cao, Liu, Saba, Huang and Bie. This is an open-access article distributed under the terms of the Creative Commons Attribution License (CC BY). The use, distribution or reproduction in other forums is permitted, provided the original author(s) or licensor are credited and that the original publication in this journal is cited, in accordance with accepted academic practice. No use, distribution or reproduction is permitted which does not comply with these terms. 